\title{
CORRELAÇÃO ENTRE OS ACHADOS CLÍNICOS DA DEGLUTIÇÃO E OS ACHADOS DA TOMOGRAFIA COMPUTADORIZADA DE CRÂNIO EM PACIENTES COM ACIDENTE VASCULAR CEREBRAL ISQUÊMICO NA FASE AGUDA DA DOENÇA
}

\author{
Anna Flávia Ferraz Barros', Soraia Ramos Cabette Fábio², Ana Maria Furkim³
}

\begin{abstract}
RESUMO - O acidente vascular cerebral (AVC) é uma das principais causas de lesões permanentes em adultos, podendo provocar seqüelas motoras globais, alterações de fala, linguagem e deglutição. Durante a fase aguda, a detecção do risco de aspiração é fundamental para prevenir complicações pulmonares e permitir apropriadas intervenções terapêuticas, possibilitando alimentação por via oral precoce e segura. Na literatura, as correlações entre o distúrbio da deglutição e a localização da lesão em pacientes com AVC são inespecíficas. Desta forma, o objetivo do presente estudo foi determinar se existe correlação entre a localização das lesão vascular encefálica e a dificuldade de deglutição em pacientes com AVC isquêmico (AVCl). Foram incluídos 27 pacientes com $\mathrm{AVCl}$ que foram submetidos à avaliação clínica da deglutição no leito. Os resultados foram confrontados com resultados obtidos pela tomografia computadorizada do crânio, relacionados à localização das lesões. Na avaliação clínica, $48 \%$ dos pacientes apresentaram disfagia orofaríngea e $52 \%$ deglutição funcional. Em $84 \%$ dos pacientes disfágicos foram observadas alterações no território carotídeo, sendo $76 \%$ na artéria cerebral média (ACM). Nos pacientes com deglutição funcional $57 \%$ apresentaram alterações em ACM e $22 \%$ em artéria cerebral posterior. O hemisfério esquerdo foi afetado em $50 \%$ dos pacientes com deglutição funcional e em $46 \%$ dos disfágicos. Em conclusão, a localização hemisférica não está associada com a presença ou não de disfagia, porém a maioria dos pacientes disfágicos apresentou alterações no território carotídeo, notadamente na ACM.
\end{abstract}

PALAVRAS-CHAVE: deglutição, disfagia, acidente vascular cerebral.

\begin{abstract}
Relation between clinical evaluation of deglutition and the computed tomography in acute ischemic stroke patients

ABSTRACT - Stroke is one of the main causes of permanent lesions in adults and can provoke global motor sequels, speech and language alterations, and swallowing. During the acute phase, the detection of aspiration risks is essential to prevent lung complications and to allow appropriate therapeutic interventions, making possible precocious oral feeding. In the literature, the correlations between the disturbance of the deglutition and the location of the lesion in patients with stroke are not specific. This way, the objective of the present study was to determine if correlation exists between the location of the vascular lesion and dysphagia in acute ischemic stroke patients. Bedside clinical evaluation of deglutition was made in 27 patients with acute ischemic stroke and the results were compared with the computed tomography findings. In the clinical evaluation, $48 \%$ patients were dysphagic and $52 \%$ had functional deglutition. In dysphagic patients, $84 \%$ had lesion in carotid territory, with $76 \%$ in the middle cerebral artery. In patients with functional deglutition, $57 \%$ had lesion in the middle cerebral artery and $22 \%$ in the posterior cerebral artery. In $50 \%$ of the patients with functional deglutition and in $46 \%$ of the dysphagics the lesion was in the left hemisphere. In conclusion, the hemispherical location is not associated with the presence or not of dysphagia, however most of the dysphagic patients presented alterations in the carotid territory, especially in the middle cerebral artery.
\end{abstract}

KEY WORDS: dysphagia, deglutition disorders, stroke.

\footnotetext{
Centro de Especialização em Fonoaudiologia Clínica (CEPAC) e Hospital das Clínicas da Faculdade de Medicina de Ribeirão Preto da Universidade de São Paulo, Ribeirão Preto SP, Brasil (HC-FMRP/USP): ${ }^{1}$ Fonoaudióloga Especializanda em Motricidade Oral com enfoque em Disfagia e Âmbito Hospitalar pelo CEFAC e Mestranda em Ciências Médicas pela FMRP/USP; ${ }^{2}$ Mestre em Neurologia, Médica Assistente em Neurologia, Coordenadora do Setor de Doenças Cerebrovasculares, HC-FMRP/USP; ${ }^{3}$ Especialista em Motricidade Oral, Mestre em Distúrbios da Comunicação pela UNIFESP-EPM, Doutora em Distúrbios da Comunicação pela UNIFESP-EPM, Diretora do Instituto de Gerenciamento em Fonoaudiologia e Deglutição (IGD), Coordenadora do Curso de Especialização em Motricidade Oral com enfoque em Disfagia no âmbito Hospitalar.
}

Recebido 24 Abril 2006, recebido na forma final 30 Junho 2006. Aceito 8 Agosto 2006.

Dra. Anna Flávia Ferraz Barros - Rua Francisco Octávio Pacca 117 - 14095-090 Ribeirão Preto SP - Brasil. E-mail: afbfono@yahoo.com.br 
O acidente vascular cerebral (AVC) é uma das principais causas de lesões permanentes (seqüelas, incapacidades) em adultos. É definido como um déficit ne u rológico (sinal e/ou sintoma) causado por interrupção do fluxo sangüíneo a uma determinada região encefálica, com duração dos sintomas maior que 24 hs e/ou presença de lesão cerebral pelos exames de imagem ${ }^{1}$. Tanto nos AVCs hemorrágicos quanto nos infartos cerebrais, as características clínicas são determinadas pela localização e extensão do prejuízo c e rebral. Anátomo-fisiologicamente, o fluxo sanguíneo cerebral é suprido por dois principais sistemas vasculares: o sistema carotídeo - artérias carótidas internas $(\mathrm{ACl})$, artérias cerebrais médias ( $\mathrm{ACM}$ ) e artérias cerebrais anteriores ( $A C A)$; e o sistema vértebrobasilar - artérias vertebrais (AV), artéria basilar ( $A B$ ) e artérias cerebrais posteriores $(A C P)^{2}$. Os comprometimentos neurológicos conseqüentes ao AVC podem resultar em seqüelas motoras globais, alterações de fala, linguagem e deglutição ${ }^{3}$.

Assim, a disfagia orofaríngea é comum em pacientes com AVC, ocorrendo em $45-65 \%$ dos $\operatorname{casos}^{4,5}$, podendo predizer morte e incapacidade ${ }^{4}$. As complicações mais comuns que advêm da disfagia são queda no estado nutricional e pneumonia aspirativa, podendo resultar em morte, principalmente em pacientes idosos ${ }^{6-8}$. Portanto, a detecção e caracterização da aspiração, que ocorre na fase faríngea são primordiais para o prognóstico e reabilitação ${ }^{9}$. Apesar de alguns pacientes apresentarem recuperação espontânea da disfagia alguns dias após o AVC, é necessário detectar o risco de aspiração durante a fase aguda, para prevenir complicações pulmonares e permitir a propriadas intervenções terapêuticas, possibilitando alimentação por via oral precoce e segura ${ }^{7}$. As disfagias orofaríngeas podem ser detectadas por meio de avaliação clínica, seguida de exames objetivos como a videofluoroscopia ou nasofibrolaringoscopia ${ }^{6}$. Estes exames estudam a dinâmica da deglutição, podendo esclarecer objetivamente os distúrbios específicos nas fases oral e/ou faríngea. Outros exames complementares são imprescindíveis em pacientes com sintomas de AVC, para estabelecer o diagnóstico etiológico e diferencial, como a tomografia computadorizada do crânio (TC) e/ou a ressonância magnética do crânio (RM), entre outros ${ }^{3}$. Utilizando-se a RM funcional em adultos saudáveis, observou-se que durante a deglutição, ocorre ativação de áreas corticais e subcorticais, sendo a mais ativada o córtex motor primário e somato-sensorial, além de outras áreas, como có rtex motor suplementar, córtex insular e o tálamo.
Tais achados sugerem que o sistema nervoso central deve utilizar diferentes estratégias funcionais para executar diferentes tipos de deglutição (deglutição seca e com bolo alimentar, por exemplo) ${ }^{10}$. Apesar de não existir localização cortical específica para o controle da deglutição, esta deve ter dominância hemisférica ${ }^{11}$. Desta forma, há estudos em pacientes pós AVC que correlacionam os distúrbios da deglutição com a localização da lesão, o que não é confirmado por outros estudos ${ }^{12-14}$. Portanto, esta correlação ainda não está totalmente estabelecida.

Smithard e col. ${ }^{15}$ estudaram pacientes durante a fase aguda (dois dias após o AVC) e subaguda (29 dias depois). Durante a fase aguda não se conseguiu estabelecer uma associação da disfagia com localização, tamanho da lesão ou número de lesões (única ou múltiplas). Na fase subaguda, de 9 pacientes que aspiraram na videofluoroscopia, 7 tinham lesões no hemisfério direito. Em pesquisa que correlacionou os achados da avaliação clínica da deglutição à beira do leito com os achados da TC em pacientes com infarto cerebral em fase crônica, verificou-se que lesões em substância branca, dilatação ventricular e graves atrofias corticais, como múltiplos infartos em hemisférios bilaterais, tratavam-se de achados relacionados à disfagia, por provável envolvimento dos tratos piramidais e extra-piramidais ${ }^{16}$. A disfagia orofaríngea grave pode ocorrer em pacientes com AVC unilateral com alteração no córtex frontal e insular, observados pela TC ou RM ${ }^{17}$. Porém, de acordo com a correlação anátomo-clínica, em pacientes com disfagia orofaríngea, o tamanho da lesão é mais importante do que a localização desta ${ }^{4}$. Através da estimulação transcraniana magnética pode-se observar que pacientes com AVC unilateral que se encontram disfágicos apre sentam menores representações faríngeas no hemisfério não afetado do que os pacientes sem alterações de deglutição, independentemente da profundidade da lesão (cortical ou subcortical). Estes achados indicam assimetria inter-hemisférica da função motora da deglutição sugerindo que, lesões no hemisfério com centro dominante da deglutição, resultam mais frequentemente em disfagia orofaríngea ${ }^{18}$. Assim, a recuperação da deglutição em pacientes disfágicos após AVC está associada com o aumento da representação faríngea no hemisfério não afetado, sugerindo a possibilidade de re o rganização deste hemisfério ${ }^{12}$.

Diante dos dados conflitantes da literatura, o objetivo do presente estudo é observar se existe correlação, nos nossos pacientes com AVC, de localização das lesões com a presença de disfagia. 


\section{MÉTODO}

A pesquisa foi realizada na Unidade de Emergência do Hospital das Clínicas da Faculdade de Medicina de Ribeirão P reto - USP (HC-FMRP/USP), no setor de Neurologia. Este trabalho foi analisado e aprovada pelo Comitê de Ética em Pesquisa do HC-FMRP/USP.

Foram incluídos pacientes adultos (acima de 18 anos) com história de primeiro acidente vascular cerebral isquêmico ( $\mathrm{AVCl}$ ) na fase aguda da doença (entre o $2^{\circ}$ e $9^{\circ}$ dia após AVCI). Foram excluídos os pacientes que apresentaram quaisquer outras alterações neurológicas ou estruturais que pudessem interferir no processo de deglutição.

Primeiramente, foram extraídos os dados demográficos, história clínica, antecedentes familiares e antecedentes pessoais da ficha de atendimento ao AVC e prontuários dos pacientes. Em seguida, foram utilizados os dados colhidos na avaliação clínica fonoaudiológica à beira do leito, solicitada de rotina pelo médico responsável. Esta constou de avaliação estrutural e funcional da deglutição, utilizandose, conforme aceitação do paciente, a consistência líquida, pastosa e sólida, observadas na dieta geral [Acesso ao protocolo de avaliação clínica: afbfono@yahoo.com.br].

Os pacientes foram então separados em dois grupos:

I - Pacientes com deglutição funcional - pacientes sem alterações de deglutição ou que apresentaram somente alterações em fase oral, sem sinais clínicos de penetração e/ou aspiração laringotraqueal do alimento na avaliação clínica.

II - Pacientes com disfagia orofaríngea neu rogênica pacientes que apresentaram sinais clínicos de penetração e/ou as piração laringotraqueal do alimento na avaliação clínica.

Os resultados dessas avaliações foram confrontados com a TC, que é realizada de rotina nas primeiras 24 horas. Nos casos em que a imagem isquêmica não foi visualizada nesse período, os pacientes foram submetidos a novo exame com 48 horas ou mais.

O resultado desses exames foi analisado em conjunto com uma neurologista especializada em doenças cerebrovasculares, observando-se a localização e extensão da lesão, artéria cerebral envolvida e presença de lesões antigas de acordo com a classificação de Goldstein e col. ${ }^{19}$. Em dois casos o exame analisado foi a RM, pois a TC não foi realizada nestes pacientes.

Para verificar possíveis diferenças entre os dois grupos, foi realizada análise estatística com a aplicação do Teste de Mann-Whitney, utilizando o programa SPSS (Statistical Package for Social Sciences), em sua versão 10.0, adotandose nível de significância de $5 \%(0,050)$.

\section{RESULTADOS}

Participaram da pesquisa 27 pacientes, com média de idade de 60 anos, sendo 15 (56\%) do sexo masculino e $12(44 \%)$ do sexo feminino.

De acordo com a avaliação clínica fonoaudiológica à beira do leito, solicitada de rotina pelo médico responsável, 14 pacientes (56\%) apresentaram deglutição funcional e 13 (48\%) disfagia orofaríngea neu-

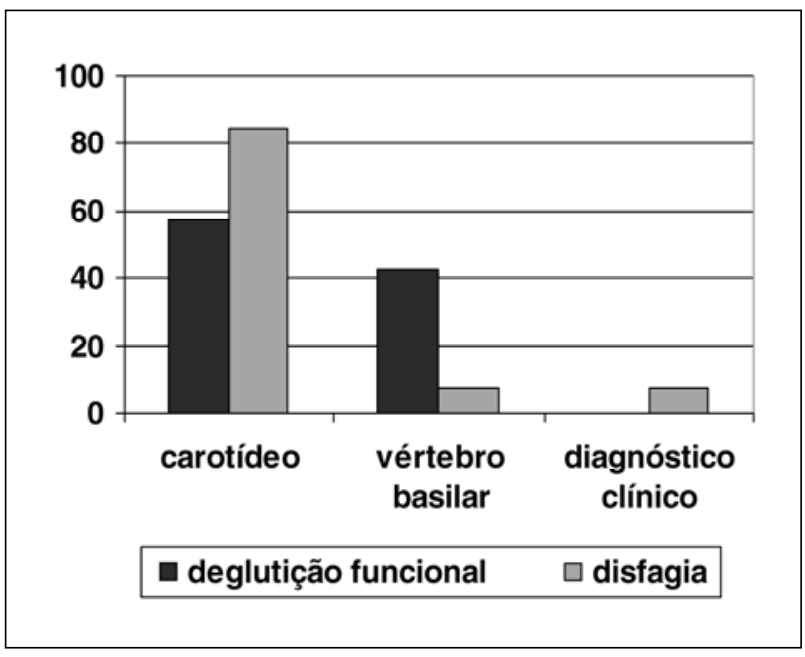

Fig 1. Correlação entre território afetado no AVC e a presença de disfagia e deglutição funcional.

rogênica. A presença de disfagia foi observada com maior freqüência no sexo feminino (62\%).

As variáveis analisadas apresentam semelhanças estatísticas entre os dois grupos considerados, porém, devido ao número pequeno de pacientes avaliados, os resultados podem apenas sugerir evidências.

Considerando-se o território afetado na análise das TC, verificou-se que tanto o grupo I (deglutição funcional) quanto o II (disfagia) apresentaram mais alterações no território carotídeo, $57 \%$ e $84 \%$ respectivamente. $O$ território vért e $b$ ro-basilar foi mais afetado nos pacientes com deglutição funcional (43\%), porém não foi considerado estatisticamente significante $(p=0,180)$ (Fig 1).

Em relação à localização específica, o território de ACM foi o mais envolvido, em $76 \%$ nos pacientes disfágicos e $57 \%$ nos pacientes com deglutição funcional, porém também não foi considerado estatisticamente significante $(p=0,095$ e $p=0,156)$. No gru $p$ I também foram observadas alterações na ACP (22\%), no tronco (14\%) e no cerebelo (7\%). No grupo II as demais alterações foram $8 \%$ na ACM e ACA, $8 \%$ na ACP e cerebelo e $8 \%$ a localização não foi visualizada pelo exame, que foi considerado normal (o AVC destes pacientes foi diagnosticado clinicamente, podendo ser decorrente de síndrome lacunar ou tronco cerebral) (Fig 2).

O hemisfério esquerdo foi afetado em $50 \%$ dos pacientes com deglutição funcional e em $46 \%$ dos disfágicos ( $p>0,999$ e $p=0,688)$.

$\mathrm{Na}$ análise dos exames de TC, de acordo com a classificação de Goldstein e col. ${ }^{19}$, observou-se que a maioria dos pacientes apresentou infarto maior que $1,5 \mathrm{~cm}$ em estruturas subcorticais no hemisfério cere- 


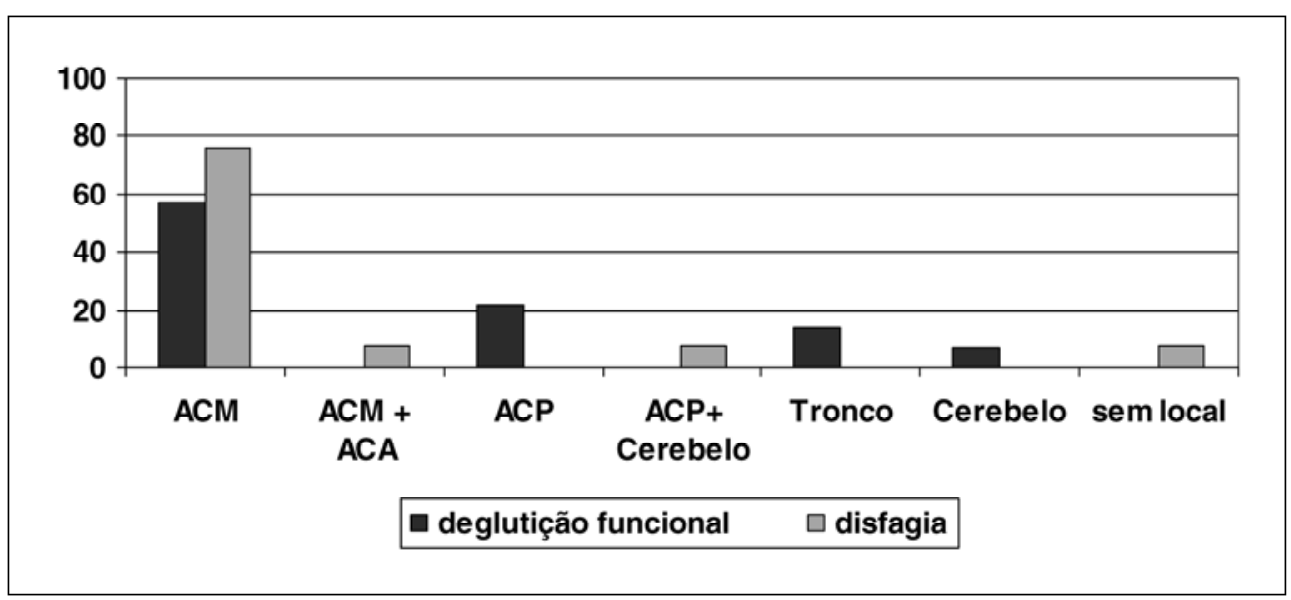

Fig 2. Correlação entre localização específica do AVC e a presença de disfagia e deglutição funcional.

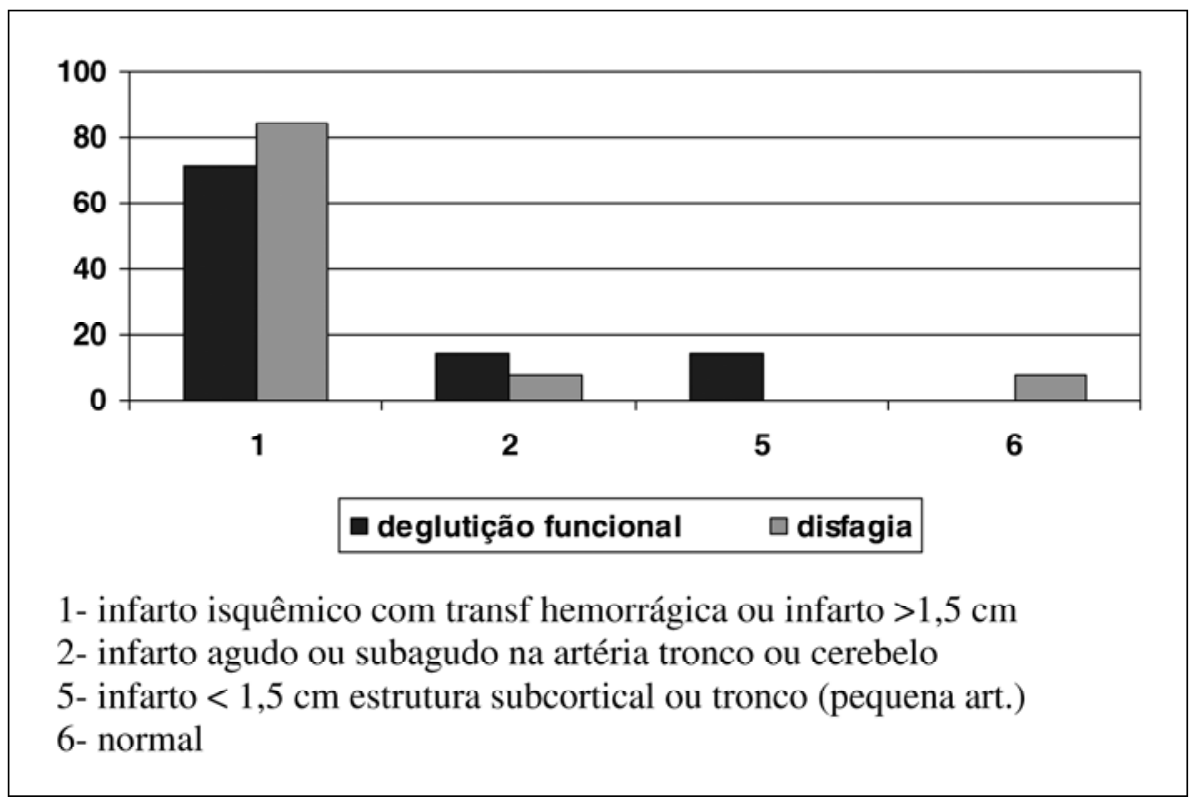

Fig 3. Correlação entre análise do exame de TC e a presença de disfagia e deglutição funcional.

bral ou infarto isquêmico com transformação hemorrágica envolvendo estruturas corticais e possivelmente se estendendo para estruturas subcorticais $(72 \%$ no grupo I e $84 \%$ no grupo II). Infarto agudo ou subagudo na distribuição de uma artéria circunferencial no t ronco cerebral e/ou cerebelo foi observado em $14 \%$ dos pacientes do grupo I e $8 \%$ do grupo II. Dois pacientes $(14 \%)$ do grupo I apresentaram infarto menor que $1,5 \mathrm{~cm}$ de diâmetro em estrutura subcortical ou tronco cerebral no território de uma pequena artéria penetrante. E um paciente (8\%) do grupo II apresentou TC normal. Os dados, porém não foram considerados estatisticamente significantes $(p=0,463)$ (Fig 3$)$.

\section{DISCUSSÃO}

No presente estudo os pacientes foram classifica- dos como apresentando deglutição funcional ou disfagia orofaríngea, utilizando-se apenas a avaliação clínica da deglutição à beira do leito, realizada por uma fonoaudióloga, não sendo utilizada avaliação instrumental, como videofluoroscopia ou nasofibroscopia. Isto porque os pacientes foram avaliados durante a fase aguda da doença (entre o $2^{\circ}$ e $9^{\circ}$ dia após $\mathrm{AVCl}$ ) não estando disponíveis os exames ao leito. Apesar desta avaliação clínica fonoaudiológica apresentar limitações s,20-22 $^{7}$ é considerada soberana em relação aos exames objetivos ${ }^{23}$.

No presente estudo $48 \%$ dos pacientes foram diagnosticados com disfagia orofaríngea neurogênica durante a fase aguda do AVC, o que corrobora a incidência encontrada em pesquisas prévias ${ }^{4,5}$. Martino e col. ${ }^{24}$ analisaram vários estudos que envolviam 
pacientes com AVC, e verificaram que independente da localização da lesão, a incidência de disfagia era baixa quando utilizada triagem para o diagnóstico de deglutição (37\%-45\%), aumentava quando este diagnóstico era por uma avaliação clínica (51\%-55\%) e passava para $64 \%-78 \%$ quando algum exame instrumental era realizado.

Assim, a grande incidência de disfagia na fase aguda do AVC reforça a necessidade de detectar o risco de aspiração durante esta fase, para prevenir complicações pulmonares e permitir apropriadas intervenções terapêuticas, possibilitando alimentação por via oral precoce e segura ${ }^{7}$.

A presença de disfagia foi observada com maior freqüência no sexo feminino (62\%), o que corrobora estudo prévio que demonstra maior gravidade, mortalidade e morbidade em mulheres que sofreram o primeiro AVC ${ }^{25}$. Porém Teasell e col. ${ }^{26}$ ao analisarem pacientes com AVC, verificaram ausência de diferença significativa quanto à idade, gênero e tipo de AVC em pacientes com e sem dificuldade de deglutição. De acordo com as pesquisas realizadas observa-se ausência de correlação específica entre a localização da lesão e a presença de disfagia em pacientes com $\mathrm{AVC}^{12-14}$. No atual estudo, apesar do número pequeno de pacientes e da análise estatística não ser significante, pode-se observar uma porcentagem maior de pacientes disfágicos com alterações no território carotídeo (84\%), especificamente na ACM (76\%), concordando com pesquisa realizada por Paciaroni e col. ${ }^{4}$, foi observada em que a presença de disfagia orofaríngea, que estava mais frequentemente associada com o envolvimento total desta artéria. Infartos que acometem a ACM (território de circulação anterior) são responsáveis pelo desenvolvimento de disfagia, pois afetam áreas rep resentativas da deglutição como tálamo, cápsula interna, região sub-insular, dentre outras áreas subcorticais ${ }^{4}$. Estudos prévios também observaram que a disfagia orofaríngea era mais prevalente em infartos com lesões grandes da circulação anterior e em AVC hemorrágico do que em lesões parciais da circulação anterior ou poste$\operatorname{rior}^{27,28}$. E que o aumento da gravidade dos pacientes com AVC na circulação anterior comparado com outros subtipos de AVC pode ser decorrente do tamanho da lesão ${ }^{28}$.

Paciaroni e col. ${ }^{4}$ inferem que pequenas lesões subcorticais $(<1,5 \mathrm{~cm})$ na circulação anterior e pequenas lesões na ACP estavam mais freqüentemente presentes em pacientes não disfágicos. No atual estudo, $14 \%$ dos pacientes com deglutição funcional apresen- taram infarto menor que $1,5 \mathrm{~cm}$ de diâmetro em estrutura subcortical ou tronco cerebral no território de uma pequena artéria penetrante, e em $22 \%$ a artéria afetada foi a posterior.

Em um paciente com disfagia orofaríngea o exame de TC foi considerado normal, sendo que o diagnóstico do AVC foi realizado clinicamente, podendo ser decorrente de síndrome lacunar ou tronco cerebral não observado no exame analisado. Sabe-se que, para revelar a presença de infarto especialmente na fossa craniana posterior, o exame de RM pode ser mais sensível que a $\mathrm{TC}^{3}$, porém não foi realizada nesse paciente. Lawrence e col. ${ }^{28}$ verificaram que os pacientes com AVC lacunar apresentavam alta prevalência de fraqueza nos membros, porém as alterações como disfagia, incontinência e disfunção cognitiva eram menos observadas.

Dois pacientes (14\%) do grupo I apresentaram alterações em tronco cerebral e ausência de dificuldade de deglutição, o que pode ser justificado pelo fato desses pacientes terem apresentado infarto menor que $1,5 \mathrm{~cm}$ de diâmetro de acordo com a classificação de Goldstein e col. ${ }^{19}$.

Lesões no tronco cerebral afetam gravemente o processo de deglutição ${ }^{24,29}$, porém são menos comuns ${ }^{24}$. O fato de nenhum paciente disfágico do estudo ter apresentado AVC de tronco, confirmado pela TC, pode ser decorrente ao número pequeno da amostra. Além disso, estudos prévios mostram que a disfagia grave e persistente é mais freqüente em pacientes com AVC de tronco cerebral e múltiplos $A V C{ }^{14,29}$. Neste estudo foram incluídos somente sujeitos com o primeiro AVC, podendo reduzir a quantidade de pacientes disfágicos pós AVC na fase aguda.

Não foi observada diferença significante entre os hemisférios esquerdo e direito nos pacientes avaliados. Em estudo realizado por Paciaroni e col. ${ }^{4}$ verificou-se que, apesar de não ocorrer diferença significativa entre os hemisférios direito e esquerdo e a p resença de disfagia, os pacientes que tiveram envolvimento da ACM e não apresentaram disfagia, a localização do AVC estava no hemisfério direito. O que discorch com outra pesquisa na qual se verificou que pacientes com AVC em território anterior da ACM di reita apresentam pior padrão de deglutição do que os pacientes com AVC em território posterior esquerdo dessa mesma artéria ${ }^{13}$.

Em estudo realizado por Hamdy e col. ${ }^{30}$, encont rou-se que a deglutição tem re $p$ resentação cortical bilateral, mas esta é assimétrica, ou seja, a dominância da deglutição pode estar no hemisfério direito para 
alguns pacientes e no hemisfério esquerdo para outros. Assim, a recuperação da deglutição em pacientes disfágicos após AVC está associada com o aumento da representação faríngea no hemisfério não afetado, sugerindo a possibilidade de reorganização deste hemisfério ${ }^{12}$.

Esta pode ser a explicação porque, apesar de mesma localização, alguns pacientes desenvolvem disfagia e outros não ${ }^{30}$. Além disso, o tamanho da lesão é considerado mais importante do que a sua localização para o desenvolvimento de disfagia orofaríngea ${ }^{4}$.

Em conclusão, há evidências de que a localização hemisférica não esteja associada com a presença ou não de disfagia, apesar da maioria dos pacientes disfágicos apre se nt a rem alterações no território caro tídeo e na artéria cerebral média. Independentemente da localização do AVC, a incidência de disfagia na fase aguda da doença foi alta, reforçando a necessidade de avaliação clínica da deglutição por um especialista na área, já que existem evidências de que a detecção da disfagia nesses pacientes reduz não apenas as complicações, mas também o tempo e o gasto com a internação ${ }^{21}$.

\section{REFERÊNCIAS}

1. Dini LI. Acidente vascular encefálico. Web: Claasic Life Medicine, Ed3 2002.

2. Whisnant JP, Basford JR, Bernstein EF, et al. Classification of cereb rovascular diseases: III. Special report from the National Institute of Neurological Disorders and Stroke. Stroke 1990;21:637-676.

3. Kelly RE. Afecções de vasos cerebrais. In Weiner WJ, Goetz CG (eds). Neurologia para o não-especialista: fundamentos básicos de neurologia contemporânea, $4^{a}$ ed. São Paulo: Livraria Santos, 2003:69-83.

4. Pacia roni M, Mazzotta G, Corea F, et al. Dysphagia following stroke. Eur Neurol 2004;51:162-167.

5. Daniels SK, Brailey K, Priestly DH, Herrington LR, Weisberg LA Founds AL. Aspiration in patients with acute stroke. A rch Phys Med Rehabil 1998;79:14-19.

6. Doggett DL, Tappe KA, Mitchel MD, Chapell R, Coates V, Turkelson $\mathrm{CM}$. Prevention in elderly stroke patients by systematic diagnosis and treatment of dysphagia: an evidence-based comprehensive analysis of the literature. Dysphagia 2001;16:279-295.

7. Leder SB, Espinosa JF. Aspiration risk after acute stroke: comparison of clinical examination and fibroscopic evaluation of swallowing. Dysphagia 2002;17:214-218.
8. Smitha rdDG, O'Neill PA, Parks C, Morris J. Complications and outcome after acute stroke: does dysphagia matter? Stroke 1996;27:12001204.

9. Furkim AM, Behlau MS, Weckx LLM. Avaliação clínica e videofluoro scópica da deglutição em crianças com paralisia cerebral tetraparética espástica. Arq Neuropsiquiatr 2003;61:611-616.

10. Mosier K, Patel R, Liu WC, Kalnin A, Maldjian J, Bareds S. Cortical representation of swallowing in normals adults: functional implications. Laringoscope 1999;109:1417-1423.

11. Smithard DG. Swallowing and stroke. Cerebrovasc Dis 2002;14:1-8.

12. Hamdy S, Aziz Q, Rothwell JC, et al. Recovery of swallowing after dysphagic stroke relates to functional reorganization in the intact motor cortex. Gastroenterology 1998;115:1104-1112.

13. Robbins J, Levine RL, Maser A, Rosenbek JC, Kempster GB. Swallowing after unilateral stroke of cerebral cortex. A rdh Phys Med Rehabil 1993; 74:1295-1300.

14. SmithardDG, O'Neill PA, England RE, et al. The natural history of dysphagia following a stroke. Dysphagia 1997;12:188-193.

15. Smithard DG, O'Neill PA, Martin DF, England RE. Aspiration following stroke: is it related to the side of stroke. Cerebrovasc Dis 2002;14:1-8.

16. Iwamoto T, Koshibu J, Kikawada M, et al. Findings of bedside swallwing assesment and brain computerized tomography in patients with chronic cerebral infarction, and their outcome (abstract). Nippon Ronen Igakkai Zasshi 2001;38:651-658.

17. Broadley S, Croser D, Cottrel J, et al. Predictors of prolonged dysphagia following acute stroke. J Clin Neuroci 2003;10:300-305.

18. Hamdy S, Aziz Q, Rothwell JC, et al. Explaining oropharyngeal dysphagia after unilateral hemispheric stroke. Lancet 1997;350:686-692.

19. Goldstein LB. Improving the reliability of stroke subgroup classification. Stroke 2001;32:1091-1097.

20. B roniatowski M. Fiberoptic endoscopic evaluation of dysphagia and videofluoroscopy. Dysphagia 1998;13:22-23.

21. Martino R, Pron G, Diamant N. Screening for oropharyngeal dysphagia in stroke: insufficient evidence for guidelines. Dysphagia 2000;15: 19-30.

22. Ramsey DJC, Smithard DG, Kalra LK. Early assesment of dysphagia and aspiration risk in acute stroke patients. Stroke 2003;34:1252-1257.

23. Schmidt BAM, Kurlinski M. Dysphagia evaluation pratices: inconsistencies in clinical assesment and instrumental examination decisionmaking. Dysphagia 2003;18:114-125.

24. Martino R, Foley N, Bhogal, Diamant N, Speechley M, Teasell R. Dysphagia after stroke: incidence, diagnosis and pulmonary complications. Stroke 2005;36:2756-2763.

25. Rouquer J, Campello AR, Gomis M. Sex differences in first-ever acute stroke. Stroke 2003;34:1581-1585.

26. Teasell R, Foley N, Fisher J, Finestone H. The incidence, management, and complications of dysphagia in patients with medullary strokes admitted to a rehabilitation unit. Dysphagia 2002;17:115-120.

27. Daniels SK, Founds AL. The role of the insular córtex in dysphagia. Dysphagia 1997;12:146-156.

28. Law rence ES, Coshall C, Dundas R, et al. Estimates of the prevalence of acute stroke impairments and disbility in a multiethnic population. Stroke 2001;32:1279-1284.

29. Han DS, Chang YC, Lu CH, Wang TG. Comparasion of disordered swallowing patterns in patients with recurrent cortical/subcortical s t roke and first-time brainstem stroke. J Rehabil Med 2005;37:189-191.

30. Hamdy S, Rothwell JC, Aziz Q, Thompson DG. Organization and reorganization of human swallowing motor cortex: implications for recovery after stroke. Eur Neurol 2004,5:162-167. 